\title{
Trends and factors associated with early initiation of breastfeeding in Namibia: analysis of the Demographic and Health Surveys 2000-2013
}

\author{
M. N. Ndirangu ${ }^{1 *}$, S. M. Gatimu ${ }^{2}$, H. M. Mwinyi ${ }^{3}$ and D. C. Kibiwott ${ }^{4}$
}

\begin{abstract}
Background: Early initiation of breastfeeding (EIBF) lowers the risk for all-cause mortality in babies, including those with low birth weight. However, rates of neonatal mortality and delayed initiation of breastfeeding remain high in most low- and middle-income countries. This study aimed to assess the trends and factors associated with EIBF in Namibia from 2000 to 2013.

Methods: An analysis of ElBF trends was conducted using data from three Namibia Demographic Health Surveys. The present sample included singleton children younger than 2-years from $2000(n=1655), 2006-2007(n=2152)$ and 2013 ( $n=2062$ ) surveys. Descriptive statistics were used to analyse respondents' demographic, socioeconomic and obstetric characteristics. Factors associated with EIBF were assessed using univariate analysis and further evaluated using multivariable logistic regression analysis.

Results: EIBF significantly decreased from 82.5\% (confidence interval [CI]: 79.5-85.0) in 2000 to $74.9 \%$ (72.5-77.2) in 2013. Factors associated with EIBF in 2000 were urban residence (adjusted odds ratio 0.58, 95\% Cl: 0.36-0.93), poorer household wealth index $(1.82,1.05-3.17)$, lack of antenatal care $(0.14,0.03-0.81)$, small birth size $(0.38,0.24-0.63)$ and large birth size $(0.51,0.37-0.79)$. In 2013, factors associated with EIBF were maternal age of $15-19$ years $(2.28,1.22-4.24)$, vaginal delivery $(2.74,1.90-3.93)$, married mothers $(1.57,1.16-2.14)$, delivery assistance from health professionals (3.67, 1.23-10.9) and birth order of fourth or above $(1.52,1.03-2.26)$.

Conclusions: Namibia has experienced a declining trend in EIBF rates from 2000 to 2013. Factors associated with EIBF differed between 2000 and 2013. The present findings highlight the importance of continued commitment to addressing neonatal health challenges and strengthening implementation of interventions to increase ElBF in Namibia.
\end{abstract}

Keywords: Breastfeeding, Early initiation, Trends, Determinants, Namibia, Demographic health survey

\section{Background}

Globally, under half of the newborns are breastfed within an hour of delivery [1]. The proportion is even lower in the African region (44\%); this rate is "fair" according to World Health Organization (WHO) classification of early initiation of breastfeeding (EIBF) but falls below "very good" (90-100\%) [2]. The benefits of EIBF for both mother and baby are well-documented, including reduced

\footnotetext{
* Correspondence: ndiranguh.michael@gmail.com

${ }^{1}$ Division of Social Medicine and Global Health, Department of Clinical

Sciences, Lund University, Malmö, Sweden

Full list of author information is available at the end of the article
}

risk of postpartum haemorrhage [3, 4], increased motherbaby bonding, increased colonisation of the baby's enteric system by microflora $[5,6]$ and reduced neonatal mortality (including among low birth weight babies) [7, 8].

Despite these benefits, the rate of EIBF in most middleincome countries remains low, including in Namibia. For example, EIBF rates are 30.8 and $41.9 \%$ in rural and urban Nigeria respectively [9], $44.7 \%$ in Algeria and $58.7 \%$ in Kenya [10]. Low EIBF rates are associated with unskilled birth attendance [11], non-health facility [12] and caesarean deliveries $[10,13]$ and maternal complications [10]. High EIBF rates are associated with health facility delivery,

(c) The Author(s). 2018 Open Access This article is distributed under the terms of the Creative Commons Attribution 4.0 International License (http://creativecommons.org/licenses/by/4.0/), which permits unrestricted use, distribution, and reproduction in any medium, provided you give appropriate credit to the original author(s) and the source, provide a link to the Creative Commons license, and indicate if changes were made. The Creative Commons Public Domain Dedication waiver (http://creativecommons.org/publicdomain/zero/1.0/) applies to the data made available in this article, unless otherwise stated. 
large birth size, formal education, urban residence, wealthier household index, non-working mothers, higher birth order and female babies [9, 13-15].

In the early 1990s, the WHO and United Nations Children's Fund (UNICEF) launched the Baby MotherFriendly Initiative (BMFI) to support breastfeeding practices. Namibia adopted this initiative in 1992 and was among the first African countries to launch BMFI [16]. In Namibia, BMFI resulted in training healthcare professionals on breastfeeding management and promotion, certification of all 35 hospitals as baby-friendly [17] and an increase in EIBF from 52\% in 1992 to $81 \%$ in 2000 [18]. However, in recent years, Namibia has experienced changes that have posed threats to these gains. The prevalence of human immunodeficiency virus (HIV) among pregnant women receiving antenatal care increased from $4.2 \%$ in 1992 to $19.3 \%$ in 2000 , with breastfeeding contributing 30-40\% of mother-to-child transmission of HIV [19]. Namibia was also reclassified as an upper middle-income country in 2009, but rates of unemployment and poverty remain high. To date, no studies in Namibia have investigated the potential effects of these changes on child health indicators, including EIBF.

Namibia also failed to meet targets for child health specified in the Millennium Development Goals (MDGs) [20]. Recent UNICEF estimates indicate the EIBF rate declined from $81 \%$ in 2000 to $71 \%$ in 2016 [1, 20]. This downward trend and the lack of evidence on changes over time in factors associated with EIBF necessitate further investigations using nationally representative data. This study aimed to assess trends and factors associated with EIBF in Namibia from 2000 to 2013.

\section{Methods}

\section{Data sources and sample}

This study used nationally representative child datasets from the Namibia Demographic and Health Surveys (NDHS) for 2000, 2006-2007 and 2013 [18, 20, 21]. All surveys used a two-stage stratified cluster sampling design based on administrative regions and locations [18, 20, 21]. The first stage involved identification of primary sampling units and the second involved selection of households. Both stages were based on the sampling frame used in the Namibia Population and Housing Census preceding the NDHS (1991, 2001 and 2011). Individual households were selected using systematic sampling [18, 20, 21]. A trained team of interviewers using standardised pre-tested household, women's and men's questionnaires (translated into six local languages) collected data for all surveys [18, 20, 21].

This study used data for households and women aged 15-49 years from the three surveys: 6849 households and 7308 women from 2000; 9970 households and 10,352 women from 2006 to 2007; and 11,004 households and 9940 women from 2013. The household response rate was $96.9 \%$ in $2000,92.3 \%$ in $2006-2007$ and $97 \%$ in 2013; individual response rates for women were $92.4,94.7$ and $92 \%$, respectively. In the 5 years preceding each survey, 3989 (2000), 5168 (2006-2007) and 5046 (2013) participating women had given birth; 1707, 2206 and 2122 children were younger than 24 months (Additional file 1: Figure S1). The present analysis included children younger than 24 months who were singleton live births (2000, $n=1655$; 2006-2007, $n=2152$; 2013, $n=2062$ ). Detailed information on NDHS data sources, survey settings and sampling strategies have been described elsewhere $[18,20,21]$.

\section{Measures}

\section{Outcome variable}

The main study outcome was EIBF, which was assessed among children younger than 24 months. EIBF is defined as putting a newborn baby to the breast within $1 \mathrm{~h}$ of birth [22]. The NDHS assessed EIBF by asking respondents, 'How long after birth did you first put (last born child's name) to the breast?' [18, 20, 21]. Responses were categorised into those who started breastfeeding within 1-h of birth and more than 1-h after birth.

\section{Explanatory variables}

We reviewed previously published studies on factors associated with EIBF to identify potential confounders, which were classified as maternal, obstetric and childrelated factors. Maternal factors included age $(<20$ years, 20-34 years, $\geq 35$ years) $[15,23]$, marital status (never married, married/cohabiting, widowed/divorced/separated), residence (urban or rural), education (no formal, primary, secondary, tertiary), occupation and household wealth [13, 15, 24, 25]. Maternal occupation was categorised as paid work (skilled/unskilled manual work, clerical, blue collar), agriculture (paid and unpaid agricultural work) and unemployed (not working, housewives, domestic work) [25]. Household wealth was categorised in quintiles (1-poorest; 5-richest) [26], generated from wealth scores calculated using principal component analysis of household assets [27].

Obstetric factors included antenatal care (ANC) visits $(0,1-3, \geq 4$, do not remember) [3, 28], place of delivery (health facility or home/other) [3], delivery mode (vaginal or caesarean section) [29] and assistance during delivery (health professional, traditional birth attendants [TBAs], self/relatives) [24]. Child-related factors were sex [13], birth size (small, average, large) [25] and birth order $(1,2-3, \geq 4)[24]$.

\section{Statistical analysis}

Data were checked for completeness and consistency. Statistical analyses on complete cases were performed with STATA version 13.1 excluding 111 (6.7\%), 209 
(9.7\%) and 155 (7.5\%) children who had missing data on the outcome variable for 2000, 2006-2007 and 2013 surveys, respectively. We used frequencies and percentages to report sample characteristics and EIBF trends, and chi-square tests to assess associations between explanatory variables and EIBF in each survey. Simple and hierarchical multivariable logistic regression analyses were conducted to assess factors associated with EIBF in each survey. To allow comparability across the surveys, variables in all three surveys with a $p$-value $>0.25$ in univariable regression analyses were excluded from the multivariable analyses [30]. Maternal, obstetric, and child-related factors were first included in the model separately and then together in a final model. We also adjusted for the sampling weight and cluster design of the surveys [31] and reported unadjusted and adjusted odds ratios (OR) with 95\% confidence intervals (CI).

\section{Results}

\section{Respondents' characteristics}

Table 1 outlines the children's and mothers' characteristics. The proportion of teenage mothers decreased from $11.4 \%$ in 2000 to $10.8 \%$ in 2013 , and that of mothers living in urban areas increased from $33.2 \%$ in 2000 to $47.6 \%$ in 2013. Mothers with no education decreased from $13.3 \%$ in 2000 to $5.5 \%$ in 2013, and rates of secondary education increased from $49.1 \%$ in 2000 to $66.7 \%$ in 2013. The proportion of health facility deliveries increased from $76.9 \%$ in 2000 to $88.5 \%$ in 2013 . Delivery assistance from TBAs decreased from $2.2 \%$ in 2000 to $0.5 \%$ in 2013 . In addition, the proportion of mothers delivering via caesarean section increased from $12.2 \%$ in 2006-2007 to $15.6 \%$ in 2013 . The proportion of male babies decreased from $50.7 \%$ in 2000 to $48.3 \%$ in 2013 , and that of large-sized babies increased from $30.4 \%$ in 2000 to $38.6 \%$ in 2013.

\section{EIBF trends in Namibia}

Namibia experienced a decline in the EIBF rate between 2000 and 2013. The weighted percentage of babies who were breastfed within $1 \mathrm{~h}$ of birth decreased significantly from $82.5 \%$ (95\% CI: $79.5-85.0$ ) in 2000 to $74.9 \%$ (95\% CI: 72.5-77.2) in 2013. However, the change from 20062007 (72.8, 95\% CI: 70.2-75.2) to 2013 was not significant.

Table 2 illustrates EIBF rates by mother and child characteristics. In all three surveys, there were equal proportions of EIBF among male and female babies, and EIBF rates were higher among mothers who lived in urban areas, delivered vaginally and in health facilities and had four or more ANC visits. EIBF was significantly associated with birth size (2000 and 2006-2007), delivery mode (2006-2007 and 2013), and occupation and marital status (2013).
Overall, there was a significant decrease in the proportion of mothers who initiated breastfeeding early. The EIBF rate among urban mothers decreased significantly from $54.8 \%$ (95\% CI: 50.9-58.7) in 2000 to $40.1 \%$ (95\% CI: 37.4-42.8) in 2013. Similarly, the EIBF rate among married mothers decreased from $42.2 \%$ (95\% CI: 38.6-45.9) in 2000 to $35.1 \%$ (95\% CI: $32.5-37.8$ ) in 2013. There was a decrease in EIBF among working mothers from 57.4\% (95\% CI: 54.0-60.8) in 2000 to $46.3 \%$ (95\% CI: 43.5-49.2) in 2013. EIBF among mothers with a secondary education increased from $39.9 \%$ (95\% CI: 36.4-43.4) in 2000 to $50 \%$ (95\% CI: 47.1-53.0) in 2013 (Table 2).

\section{Factors associated with EIBF in Namibia}

In the bivariate analysis, EIBF was significantly associated with birth order and birth size in 2000, birth size, maternal age, and delivery mode in 2006-2007 and birth size, birth order, delivery assistance by TBAs, delivery mode, ANC, occupation, wealth, education and marital status in 2013 (Table 3).

The multivariate analysis showed that in 2000, the odds of EIBF were $82 \%$ higher among mothers in households with a poorer wealth index compared with richer households (AOR 1.82, 95\% CI: 1.05-3.17). In addition, mothers in rural areas had $42 \%$ (AOR $0.58,95 \%$ CI $0.36-0.93$ ) reduced odds of EIBF compared with urban mothers. Mothers who attended the recommended four or more ANC visits had 86\% (AOR 0.14, 95\% CI: 0.03-0.81) reduced odds of EIBF compared with those not attending ANC. In 2006-2007, the odds of EIBF were 49\% higher among mothers aged 20-34 years compared with those aged $\geq 35$ years, $53 \%$ higher among health facility deliveries compared with home deliveries and 2.58 times higher among those who had a vaginal delivery compared with a caesarean section. In 2013, mothers aged 15-19 years (AOR 2.28, 95\% CI: 1.22-4.24), married mothers (AOR 1.57, 95\% CI: 1.16-2.14) and those who had a vaginal delivery (AOR 2.74, 95\% CI: 1.90-3.93) had higher odds of EIBF compared with older women, unmarried women and those who had a caesarean section, respectively. Babies with a birth order of fourth or above had 52\% increased odds of EIBF compared with first-born babies (AOR 1.52, 95\% CI: 1.03-2.26). In 2006-2007, health professionalassisted deliveries had $81 \%$ reduced odds of EIBF compared with TBAs-assisted delivery (AOR 0.19, 95\% CI: 0.04-0.91). In contrast, the odds of EIBF among health professional-assisted deliveries in 2013 were 3.67 times higher compared with TBAs-assisted deliveries (AOR 3.67, 95\% CI: 1.23-10.9) (Table 3).

\section{Discussion}

The WHO classifies EIBF rates as poor (0-29\%), fair (30-49\%), good (50-89\%) and very good (90-100\%) [2]. The EIBF rate in Namibia is still considered good despite 
Table 1 Respondents' characteristics

\begin{tabular}{|c|c|c|c|c|c|c|}
\hline \multirow[b]{2}{*}{ Characteristics } & \multicolumn{2}{|c|}{2000} & \multicolumn{2}{|c|}{ 2006-2007 } & \multicolumn{2}{|c|}{2013} \\
\hline & $n$ & $\%$ & $n$ & $\%$ & $n$ & $\%$ \\
\hline
\end{tabular}

\begin{tabular}{cllllll}
\hline Age, years & & & & & & \\
$15-19$ & 189 & 11.4 & 252 & 11.5 & 222 & 10.8 \\
$20-34$ & 1124 & 69.0 & 1508 & 71.6 & 1436 & 71.0 \\
$35-49$ & 297 & 19.6 & 355 & 16.9 & 381 & 18.2 \\
Residence & & & & & & \\
Urban & 604 & 33.2 & 783 & 40.3 & 910 & 47.6 \\
Rural & 1006 & 66.8 & 1332 & 59.7 & 1129 & 52.4
\end{tabular}

Marital status

$\begin{array}{lllllll}\text { Never married } & 664 & 44.4 & 944 & 44.9 & 1021 & 51.3\end{array}$

Married/cohabiting

Divorced/separated/

841

widow

Education

No education
Primary
Secondary
Higher education
Occupation

Not working

Agriculture

Paid work

Wealth, quintiles

Poorest

Poorer

Average

Richer

Richest

ANC attendance, visits

0

$1-3$

$\geq 4$

Do not remember

Place of delivery

Health facility

Home/others

Delivery mode

$$
\text { Vaginal }
$$

Parity

1

$\begin{array}{llllll}268 & 13.3 & 250 & 10.7 & 155 & 5.5 \\ 555 & 35.2 & 626 & 27.9 & 470 & 22.0 \\ 751 & 49.1 & 1164 & 57.1 & 1327 & 66.7 \\ 36 & 2.4 & 75 & 4.3 & 87 & 5.7\end{array}$

$\begin{array}{llllll}1034 & 66.2 & 1052 & 49.2 & 1203 & 57.1\end{array}$

$\begin{array}{llllll}66 & 5.8 & 269 & 12.6 & 36 & 1.4\end{array}$

$\begin{array}{llllll}503 & 28.1 & 778 & 38.2 & 798 & 41.4\end{array}$

$\begin{array}{llllll}340 & 23.8 & 422 & 19.8 & 410 & 21.3\end{array}$

$\begin{array}{llllll}298 & 21.9 & 451 & 20.8 & 410 & 20.3\end{array}$

$\begin{array}{llllll}317 & 19.5 & 392 & 19.1 & 397 & 19.1\end{array}$

$\begin{array}{llllll}318 & 17.3 & 417 & 21.1 & 408 & 18.7\end{array}$

$\begin{array}{llllll}316 & 17.5 & 418 & 19.3 & 403 & 20.6\end{array}$

$\begin{array}{llllll}113 & 6.1 & 86 & 3.9 & 82 & 3.2\end{array}$

$\begin{array}{llllll}285 & 18.3 & 378 & 17.7 & 279 & 13.1\end{array}$

$\begin{array}{llllll}1031 & 68.2 & 1451 & 71.3 & 1237 & 62.5\end{array}$

$\begin{array}{llllll}138 & 7.3 & 140 & 7.1 & 390 & 21.3\end{array}$

$\begin{array}{llllll}1211 & 76.9 & 1704 & 81.9 & 1761 & 88.5\end{array}$

$\begin{array}{llllll}393 & 23.1 & 410 & 18.1 & 277 & 11.5\end{array}$

$\begin{array}{llllll}- & - & 1891 & 87.8 & 1741 & 84.4\end{array}$

$\begin{array}{llllll}- & - & 220 & 12.2 & 295 & 15.6\end{array}$

$\begin{array}{llllll}470 & 29.9 & 666 & 32.0 & 625 & 31.7\end{array}$

$\begin{array}{llllll}630 & 38.3 & 904 & 43.0 & 860 & 42.7\end{array}$

$\begin{array}{llllll}510 & 31.9 & 545 & 25.0 & 554 & 25.6\end{array}$

\begin{tabular}{|c|c|c|c|c|c|c|}
\hline \multirow[b]{2}{*}{ Characteristics } & \multicolumn{2}{|l|}{2000} & \multicolumn{2}{|c|}{$2006-2007$} & \multicolumn{2}{|l|}{2013} \\
\hline & $n$ & $\%$ & $n$ & $\%$ & $n$ & $\%$ \\
\hline \multicolumn{7}{|c|}{ Assistance during labour } \\
\hline Health professional & 1396 & 90.0 & 1944 & 94.5 & 1896 & 96.2 \\
\hline TBAs & 32 & 2.2 & 28 & 1.3 & 15 & 0.5 \\
\hline Relatives/self & 136 & 7.8 & 94 & 4.2 & 84 & 3.3 \\
\hline \multicolumn{7}{|l|}{ Birth order } \\
\hline 1 & 482 & 30.4 & 683 & 32.6 & 643 & 32.5 \\
\hline $2-3$ & 622 & 38.0 & 890 & 42.5 & 845 & 42.1 \\
\hline $4+$ & 506 & 31.6 & 542 & 24.9 & 551 & 25.5 \\
\hline \multicolumn{7}{|l|}{ Baby's sex } \\
\hline Male & 807 & 50.7 & 1104 & 52.1 & 990 & 48.3 \\
\hline Female & 803 & 49.3 & 1011 & 47.9 & 1049 & 51.7 \\
\hline \multicolumn{7}{|l|}{ Baby's birth size } \\
\hline Large & 464 & 30.4 & 739 & 35.7 & 779 & 38.6 \\
\hline Average & 844 & 53.1 & 1033 & 48.5 & 886 & 43.3 \\
\hline Small & 291 & 16.5 & 337 & 15.7 & 392 & 18.1 \\
\hline
\end{tabular}

Table 1 Respondents' characteristics (Continued)

the significant decline from $82.5 \%$ in 2000 to $74.9 \%$ in 2013. This trend is similar to those witnessed in other middle-income countries such as Vietnam (62\%) and Haiti (69\%) [29, 32], and in lower-middle income settings (82\%) [33-35]. The decline in the EIBF rate in Namibia between 2000 and 2006-2007 may be attributed to the high rates of HIV [19], insufficient health infrastructure, poor access, and ineffective and inefficient health service provision [36]. However, the slight increase in the EIBF rate between the 2006-2007 and 2013 surveys may be explained by government efforts, such as enactment of infant and young child feeding policies [36].

Our findings showed the number and nature of factors associated with EIBF varied from 2000 to 2013. Overall, this may be attributable to sociocultural and economic changes, increased rural-urban migration, improved school enrolment among girls, reduced teenage pregnancies, increased employment among women and health service use [37]. We found significant associations between EIBF and delivery mode, ANC attendance, baby's birth size, place of residence, maternal age, marital status, delivery assistance and birth order. EIBF was also more likely among mothers who had a vaginal delivery compared with a caesarean section [13, 33, 38-40]. This may be partly explained by the increased rates of caesarean sections in 2006-2007 and 2013, effects of anaesthesia delaying the onset of lactation, associated respiratory distress among babies delivered by caesarean section [15] and healthcare professionals' increased preoccupation with assisting mothers to stabilise rather than initiating breastfeeding $[11,41]$. This highlights the need for 
Table 2 Rates of early initiation of breastfeeding in Namibia (2000, 2006-2007 and 2013) by demographic and socioeconomic characteristics

\begin{tabular}{|c|c|c|c|c|c|c|c|c|c|c|c|c|}
\hline \multirow[t]{2}{*}{ Characteristics } & \multicolumn{3}{|l|}{2000} & \multicolumn{3}{|c|}{ 2006-2007 } & \multicolumn{3}{|c|}{2013} & \multicolumn{3}{|c|}{ Percentage difference } \\
\hline & $\%$ & $95 \% \mathrm{Cl}$ & $p$-value ${ }^{a}$ & $\%$ & $95 \% \mathrm{Cl}$ & $p$-value ${ }^{a}$ & $\%$ & $95 \% \mathrm{Cl}$ & $p$-value ${ }^{a}$ & $2000-2006^{b}$ & $2006-2013^{b}$ & $2000-2013^{b}$ \\
\hline \multicolumn{13}{|l|}{ Age, years } \\
\hline $15-19$ & 8.9 & $7.2-10.8$ & & 7.9 & $6.7-9.4$ & & 9.1 & $7.7-10.6$ & & -1 & 1.2 & 0.2 \\
\hline $20-34$ & 58.2 & $54.4-61.9$ & 0.143 & 53.1 & $50.4-55.8$ & 0.057 & 52.7 & $50.0-55.3$ & 0.160 & -5.1 & -0.4 & -5.5 \\
\hline $35-49$ & 15.4 & $13.3-17.8$ & & 11.7 & $10.1-13.6$ & & 13.2 & 11.4-15.2 & & -3.7 & 1.5 & -2.2 \\
\hline \multicolumn{13}{|l|}{ Residence } \\
\hline Rural & 27.6 & $23.8-31.7$ & 0.210 & 27.7 & $24.8-30.7$ & 0.856 & 34.9 & $32.3-37.4$ & 0.579 & 0.1 & $7.2^{*}$ & $7.3^{* *}$ \\
\hline Urban & 54.8 & $50.9-58.7$ & & 45.1 & $42.5-47.8$ & & 40.1 & $37.4-42.8$ & & -9.7 & -5 & $-14.7^{* *}$ \\
\hline \multicolumn{13}{|l|}{ Marital status } \\
\hline Never married & 35.8 & $32.3-39.4$ & & 33.2 & $30.5-35.9$ & & 37.1 & $34.4-39.7$ & & -2.6 & 3.9 & 1.3 \\
\hline Married/cohabiting & 42.2 & $38.6-45.9$ & 0.322 & 36 & $33.3-38.8$ & 0.497 & 35.1 & $32.5-37.8$ & 0.021 & -6.2 & -0.9 & -7.1 \\
\hline $\begin{array}{l}\text { Divorced/separated } \\
\text { /widow }\end{array}$ & 4.4 & $3.4-5.8$ & & 3.6 & $2.7-4.7$ & & 2.8 & $2.0-3.8$ & & -0.8 & -0.8 & -1.6 \\
\hline \multicolumn{13}{|l|}{ Education } \\
\hline No formal & 11.5 & $8.9-14.6$ & & 7.8 & $6.5-9.4$ & & 4.2 & $3.3-5.4$ & & -3.7 & $-3.6^{* *}$ & $-7.3^{* *}$ \\
\hline Primary & 29.1 & $25.6-32.8$ & 0.805 & 21.3 & $19.4-23.5$ & 0.542 & 17.3 & 15.3-19.4 & 0.071 & $-7.8^{* *}$ & -4 & $-11.8^{* *}$ \\
\hline Secondary & 39.9 & $36.4-43.4$ & & 40.6 & $37.8-43.4$ & & 50.0 & $47.1-53.0$ & & 0.7 & 9.4 & $10.1^{* *}$ \\
\hline Tertiary & 2.0 & $1.2-3.3$ & & 3.0 & $2.0-4.5$ & & 3.4 & $2.4-4.9$ & & 1 & 0.4 & 1.4 \\
\hline \multicolumn{13}{|l|}{ Occupation } \\
\hline Not working & 54.3 & $50.7-57.8$ & & 36.6 & 33.9-39.4 & & 44.2 & $41.4-47.1$ & & $-12.8^{* *}$ & 1.7 & $-11.1^{* *}$ \\
\hline Agriculture & 5.2 & $3.3-7.9$ & 0.114 & 9.4 & $7.8-11.1$ & 0.933 & 1.1 & $0.7-1.8$ & 0.023 & 3.3 & 0.3 & 3.6 \\
\hline Paid work & 23.0 & $20.2-26.0$ & & 27.0 & $24.1-30.0$ & & 29.6 & $27.3-32.0$ & & 3.3 & 0.3 & 3.6 \\
\hline \multicolumn{13}{|l|}{ Wealth, quintiles } \\
\hline Poorest & 19.9 & $17.1-23.1$ & & 15.1 & $13.1-17.2$ & & 15.7 & 13.7-18.0 & & -4.8 & 0.6 & -4.2 \\
\hline Poorer & 19.0 & $16.2-22.2$ & & 15.9 & 13.9-18.1 & & 15.8 & $13.7-18.1$ & & -3.1 & -0.1 & -3.2 \\
\hline Average & 16.0 & $13.1-19.5$ & 0.378 & 13.5 & $11.6-15.7$ & 0.624 & 15.0 & $12.9-17.2$ & 0.198 & -2.5 & 1.5 & -1 \\
\hline Richer & 13.8 & $11.2-16.8$ & & 14.5 & $12.7-16.6$ & & 14.2 & $12.3-16.4$ & & 0.7 & -0.3 & 0.4 \\
\hline Richest & 13.8 & $11.1-17.0$ & & 13.9 & $12.2-15.8$ & & 14.3 & $12.3-16.6$ & & 0.1 & 0.4 & 0.5 \\
\hline \multicolumn{13}{|l|}{ ANC attendance, visits } \\
\hline 0 & 5.4 & $4.0-7.3$ & & 3.1 & $2.3-4.3$ & & 2.6 & $1.9-3.5$ & & -2.3 & -0.5 & $-2.8^{* *}$ \\
\hline $1-3$ & 15.3 & $13.3-17.5$ & 0.523 & 13.5 & $11.9-15.2$ & 0.379 & 10.3 & $8.8-11.9$ & 0.063 & -1.8 & -3.2 & $-5^{* *}$ \\
\hline$\geq 4$ & 55.2 & $51.2-59.0$ & & 51.4 & $48.5-54.2$ & & 45.7 & $42.8-45.6$ & & -3.8 & $-5.7^{* *}$ & $-9.5^{* *}$ \\
\hline Don't remember & 6.3 & $4.7-8.3$ & & 4.8 & $3.9-5.8$ & & 16.4 & $14.2-18.8$ & & -1.5 & $11.6^{* *}$ & $10.1^{* *}$ \\
\hline \multicolumn{13}{|l|}{ Place of delivery } \\
\hline Health facility & 63.5 & $59.9-67.0$ & 0.870 & 60.0 & $57.1-62.7$ & 0.342 & 65.9 & $63.4-68.4$ & 0.456 & -3.5 & $5.9^{*}$ & 2.4 \\
\hline Home/others & 19.0 & $16.8-22.3$ & & 12.9 & $11.2-14.8$ & & 9.0 & $7.6-10.6$ & & $-6.1^{*}$ & -3.9 & $-10^{* *}$ \\
\hline \multicolumn{13}{|l|}{ Delivery mode } \\
\hline Vaginal & - & - & - & 66.9 & $64.3-69.5$ & $<0.001$ & 66.2 & $63.6-68.7$ & $<0.001$ & & -0.7 & \\
\hline Caesarean & - & - & - & 5.8 & $4.6-7.4$ & & 8.7 & $7.2-10.5$ & & & 2.9 & \\
\hline \multicolumn{13}{|l|}{ Parity } \\
\hline 1 & 23.3 & $20.9-26.0$ & & 23.6 & $21.2-26.3$ & & 23.9 & $21.8-26.2$ & & 0.3 & 0.3 & 0.6 \\
\hline $2-3$ & 33.0 & $30.4-35.7$ & 0.093 & 30.9 & $28.6-33.2$ & 0.466 & 31.3 & $29.0-33.7$ & 0.086 & -2.1 & 0.4 & -1.7 \\
\hline $4+$ & 26.1 & $23.1-29.4$ & & 18.3 & $16.3-20.5$ & & 19.7 & $17.6-22.0$ & & -7.8 & 1.4 & $-6.4^{* *}$ \\
\hline
\end{tabular}


Table 2 Rates of early initiation of breastfeeding in Namibia (2000, 2006-2007 and 2013) by demographic and socioeconomic characteristics (Continued)

\begin{tabular}{|c|c|c|c|c|c|c|c|c|c|c|c|c|}
\hline \multirow[t]{2}{*}{ Characteristics } & \multicolumn{3}{|l|}{2000} & \multicolumn{3}{|c|}{$2006-2007$} & \multicolumn{3}{|l|}{2013} & \multicolumn{3}{|c|}{ Percentage difference } \\
\hline & $\%$ & $95 \% \mathrm{Cl}$ & $\overline{p \text {-value }}{ }^{\mathrm{a}}$ & $\%$ & $95 \% \mathrm{Cl}$ & $\overline{p \text {-value }}{ }^{a}$ & $\%$ & $95 \% \mathrm{Cl}$ & $\overline{p \text {-value }}{ }^{a}$ & $2000-2006^{b}$ & $2006-2013^{b}$ & $2000-2013^{b}$ \\
\hline \multicolumn{13}{|l|}{ Delivery assistance } \\
\hline Health professional & 73.9 & $70.7-76.8$ & & 68.2 & $65.6-70.7$ & & 72.1 & $69.7-74.4$ & 0.049 & -5.7 & 3.9 & -1.8 \\
\hline TBAs & 2.0 & $1.3-3.1$ & 0.438 & 1.3 & $0.7-2.1$ & 0.062 & 0.2 & $0.1-0.5$ & & -0.7 & $-1.1^{*}$ & $-1.8^{*}$ \\
\hline Relative/self & 6.2 & $4.7-8.2$ & & 3.3 & $2.4-4.4$ & & 2.6 & $2.0-3.5$ & & -2.9 & -0.7 & $-3.6^{* *}$ \\
\hline \multicolumn{13}{|l|}{ Birth order } \\
\hline 1 & 23.8 & $21.3-26.4$ & & 23.6 & $21.2-26.3$ & & 23.9 & $21.8-26.2$ & 0.086 & -0.2 & 0.3 & 0.1 \\
\hline $2-3$ & 32.8 & $30.2-35.6$ & 0.111 & 30.9 & $28.6-33.2$ & 0.466 & 31.3 & $28.9-33.7$ & & -1.9 & 0.4 & -1.5 \\
\hline $4+$ & 25.8 & $22.8-29.1$ & & 18.3 & $16.3-20.5$ & & 19.7 & $17.6-22.0$ & & -7.5 & 1.4 & -6.1 \\
\hline \multicolumn{13}{|l|}{ Baby's sex } \\
\hline Male & 41.2 & $37.8-44.6$ & 0.426 & 36.4 & $34.0-38.9$ & 0.284 & 36.8 & $34.4-39.2$ & 0.134 & -4.8 & 0.4 & -4.4 \\
\hline Female & 41.3 & $38.3-44.3$ & & 36.4 & $33.8-39.1$ & & 38.1 & $35.7-40.6$ & & -4.9 & 1.7 & -3.2 \\
\hline \multicolumn{13}{|l|}{ Baby's birth size } \\
\hline Small & 23.5 & $20.4-26.8$ & & 24.6 & $22.2-27.2$ & & 28.6 & $26.1-31.1$ & & 1.1 & 4 & 5.1 \\
\hline Average & 47.2 & $43.5-50.9$ & 0.001 & 37.7 & $35.2-40.4$ & 0.008 & 33.6 & $31.0-36.3$ & 0.090 & $-9.5^{* *}$ & -4.1 & $-13.6^{* *}$ \\
\hline Large & 11.7 & $9.7-14.0$ & & 10.4 & $8.8-12.1$ & & 12.8 & $11.0-14.9$ & & -1.3 & 2.4 & 1.1 \\
\hline
\end{tabular}

ANC antenatal care, $\mathrm{Cl}$ confidence interval, TBAs traditional birth attendants

${ }^{a} p$-value for association for each year

${ }^{b}$ Percentage point difference between survey years with a significance tests for difference in proportions; ${ }^{*} p$-value $<0.05$; ${ }^{* *} p$-value $<0.01$

appropriate guidelines to reduce the number of caesarean deliveries and educate mothers about the negative association between pre-labour caesarean delivery decisions and implications for the baby's wellbeing.

Lower ANC attendance leading to delayed initiation of breastfeeding in 2000 was consistent with previous studies $[33,42]$. It is paramount to promote skilled birth attendance and baby-friendly initiatives in health facilities [22] and improve new mothers' breastfeeding practices through nutrition education during ANC visits [43]. Findings from Nigeria and Brazil indicate EIBF was more likely among mothers who had large babies at birth $[9,14,25,26]$. In contrast, we found that EIBF was less likely among both large- and small-sized babies between 2000 and 2006-2007 compared with average-sized babies. Small babies often have weak breastfeeding reflexes, poor coordination, and difficulty swallowing $[14,26]$. This may be attributable to healthcare providers' increased attention to stabilising the baby rather than easing the initiation of breastfeeding [26]. Existing literature also shows that both mothers and healthcare providers perceive large babies as healthy, leading to EIBF [26].

We found EIBF was more likely among urban mothers compared with rural mothers, which was consistent with previous studies [14, 44]. This may be explained by higher ANC attendance rates, increased levels of employment and higher education levels among mothers living in urban areas. Urban women may also have increased access to information, leading to higher EIBF rates [45]. However, in 2013, place of residence was no longer a significant factor. This may be because of increased urbanisation and service provision to various parts of the country [20].

Younger women and adolescents had increased odds of initiating breastfeeding within the one-hour post-delivery period, which was similar to findings from low and middle-income countries $[10,46]$. This may be attributable to improved girls education, numbers of planned pregnancies and social support [32], and the intention to breastfeed and better prenatal attitude [47, 48]. Moreover, maternal age as a determinant of EIBF is largely dependent on the presence of factors such education level and residency; in the absence of those factors, age may not impact the EIBF rate [10]. Other factors associated with EIBF included marital status and birth order. EIBF was more likely among married mothers and babies born into large families, which may be because of psychosocial support from family [49]. Multiparous women also have an increased level of knowledge and experience, and EIBF may, therefore, be more likely.

We did not find a significant association between socioeconomic status and EIBF, which was consistent with a study on trends and determinants of EIBF in Vietnam $[29,44]$. Education, occupation, and wealth were not significantly associated with EIBF, except in 2000 where women in the poorer quintile had increased odds of EIBF. This finding may show the diminishing influence of socioeconomic factors on the uptake of health 
Table 3 Univariate and multivariable logistic regression analyses of factors associated with early initiation of breastfeeding

\begin{tabular}{|c|c|c|c|c|c|c|}
\hline \multirow[b]{2}{*}{ Characteristics } & \multicolumn{2}{|l|}{2000} & \multicolumn{2}{|l|}{ 2006-2007 } & \multicolumn{2}{|l|}{2013} \\
\hline & COR $(95 \% \mathrm{Cl})$ & AOR $(95 \% \mathrm{Cl})$ & COR $(95 \% \mathrm{Cl})$ & AOR $(95 \% \mathrm{Cl})$ & COR $(95 \% \mathrm{Cl})$ & AOR $(95 \% \mathrm{Cl})$ \\
\hline \multicolumn{7}{|l|}{ Age, years } \\
\hline $15-19$ & $0.91(0.51-1.63)$ & $1.10(0.53-2.31)$ & $0.87(0.57-1.34)$ & $1.00(0.57-1.75)$ & $1.56(0.96-2.56)$ & $2.28(1.22-4.24)^{*}$ \\
\hline $20-34$ & $1.38(0.89-2.15)$ & $1.55(0.91-2.62)$ & $1.26(0.94-1.69)$ & $1.49(1.07-2.08)^{*}$ & $1.05(0.76-1.46)$ & $1.30(0.90-1.87)$ \\
\hline $35-49$ & 1.00 & 1.00 & 1.00 & 1.00 & 1.00 & 1.00 \\
\hline \multicolumn{7}{|l|}{ Residence } \\
\hline Rural & 1.00 & 1.00 & 1.00 & 1.00 & 1.00 & 1.00 \\
\hline Urban & $0.74(0.46-1.19)$ & $0.58(0.36-0.93)^{*}$ & $1.03(0.77-1.36)$ & $0.89(0.64-1.24)$ & $1.07(0.84-1.38)$ & $0.93(0.68-1.26)$ \\
\hline \multicolumn{7}{|l|}{ Marital status } \\
\hline Never married & 1.00 & 1.00 & 1.00 & 1.00 & 1.00 & 1.00 \\
\hline Married/cohabiting & $1.29(0.86-1.94)$ & $1.23(0.73-2.07)$ & $0.99(0.78-1.27)$ & $0.94(0.72-1.24)$ & $1.44(1.10-1.90)^{*}$ & $1.57(1.16-2.14)^{*}$ \\
\hline Divorced/separated & $0.93(0.47-1.81)$ & $0.96(0.47-1.96)$ & $1.44(0.78-2.64)$ & $1.41(0.75-2.65)$ & $1.09(0.59-2.01)$ & $1.11(0.60-2.03)$ \\
\hline \multicolumn{7}{|l|}{ Education } \\
\hline Tertiary & 1.00 & 1.00 & 1.00 & 1.00 & 1.00 & 1.00 \\
\hline Secondary & $0.96(0.38-2.47)$ & $0.90(0.32-2.51)$ & $1.11(0.55-2.26)$ & $0.86(0.37-1.99)$ & $1.75(0.98-3.11)$ & $1.22(0.51-2.19)$ \\
\hline Primary & $0.98(0.41-2.39)$ & $0.89(0.32-2.43)$ & $1.35(0.66-2.76)$ & $1.03(0.43-2.48)$ & $1.95(1.07-3.56)^{*}$ & $1.06(0.51-2.19)$ \\
\hline No formal & $1.23(0.46-3.23)$ & $0.95(0.31-2.88)$ & $1.28(0.62-2.67)$ & $0.93(0.37-2.38)$ & $2.31(1.14-4.68)^{*}$ & $1.21(0.51-2.86)$ \\
\hline \multicolumn{7}{|l|}{ Occupation } \\
\hline Paid work & 1.00 & 1.00 & 1.00 & 1.00 & 1.00 & 1.00 \\
\hline Agriculture & $4.03(0.98-16.5)$ & $2.87(0.69-11.9)$ & $1.01(0.71-1.43)$ & $0.91(0.62-1.35)$ & $1.62(0.62-4.24)$ & $1.55(0.58-4.11)$ \\
\hline Not working & $1.03(0.66-1.56)$ & $1.05(0.69-1.60)$ & $1.04(0.77-1.41)$ & $0.98(0.72-1.34)$ & $1.36(1.07-174)^{*}$ & $1.27(0.97-1.66)$ \\
\hline \multicolumn{7}{|l|}{ Wealth } \\
\hline Richest & 1.00 & 1.00 & 1.00 & 1.00 & 1.00 & 1.00 \\
\hline Richer & $0.87(0.56-1.35)$ & $0.91(0.54-1.51)$ & $0.90(0.63-1.29)$ & $0.87(0.60-1.26)$ & $1.51(1.04-2.02)^{*}$ & $1.35(0.90-2.03)$ \\
\hline Average & $1.30(0.81-2.10)$ & $1.52(0.86-2.68)$ & $0.94(0.68-1.30)$ & $0.93(0.65-1.33)$ & $1.47(0.98-2.20)$ & $1.22(0.78-1.91)$ \\
\hline Poorer & $1.36(0.82-2.22)$ & $1.82(1.05-3.17)^{*}$ & $1.14(0.79-1.64)$ & $1.07(0.73-1.58)$ & $1.33(0.93-1.92)$ & $1.02(0.67-1.56)$ \\
\hline Poorest & $1.13(0.70-1.81)$ & $1.52(0.89-2.57)$ & $1.13(0.76-1.68)$ & $1.08(0.70-1.66)$ & $1.26(0.86-1.85)$ & $0.94(0.59-1.48)$ \\
\hline \multicolumn{7}{|l|}{ ANC attendance, visits } \\
\hline 0 & 1.00 & 1.00 & 1.00 & 1.00 & 1.00 & 1.00 \\
\hline $1-3$ & $0.69(0.28-1.70)$ & $0.19(0.04-1.05)$ & $0.81(0.44-1.52)$ & $1.02(0.12-8.55)$ & $0.62(0.30-1.26)$ & $0.17(0.01-3.16)$ \\
\hline $4+$ & $0.57(0.25-1.29)$ & $0.14(0.03-0.81)^{*}$ & $0.67(0.37-1.20)$ & $0.85(0.10-7.09)$ & $0.45(0.24-0.84)^{*}$ & $0.14(0.01-2.51)$ \\
\hline Do not remember & $0.67(0.22-2.05)$ & $0.18(0.03-1.21)$ & $0.65(0.34-1.28)$ & $0.79(0.09-6.65)$ & $0.57(0.29-1.14)$ & $0.16(0.01-3.09)$ \\
\hline \multicolumn{7}{|l|}{ Place of delivery } \\
\hline Home/others & 1.00 & 1.00 & 1.00 & 1.00 & 1.00 & 1.00 \\
\hline Health facility & $1.03(0.70-1.53)$ & $1.33(0.86-2.07)$ & $1.13(0.87-1.47)$ & $1.53(1.10-2.14)^{*}$ & $0.88(0.63-1.23)$ & $1.25(0.83-1.90)$ \\
\hline \multicolumn{7}{|l|}{ Delivery mode ${ }^{a}$} \\
\hline Caesarean & - & - & 1.00 & 1.00 & 1.00 & 1.00 \\
\hline Vaginal & - & - & $2.53(1.75-3.68)^{* *}$ & $2.58(1.68-3.97)^{* *}$ & $2.84(2.03-3.97)^{* *}$ & $2.74(1.90-3.93)^{* * *}$ \\
\hline \multicolumn{7}{|l|}{ Delivery assistance } \\
\hline TBAs & 1.00 & 1.00 & 1.00 & 1.00 & 1.00 & 1.00 \\
\hline Health professional & $0.47(0.15-1.47)$ & $0.48(0.14-1.61)$ & $0.32(0.10-1.01)$ & $0.19(0.04-0.91)^{*}$ & $3.60(1.09-11.8)^{*}$ & $3.67(1.23-10.9)^{*}$ \\
\hline Relative/self & $0.44(0.13-1.47)$ & $0.13(0.02-0.81)$ & $0.47(0.13-1.71)$ & $0.30(0.02-4.30)$ & $5.31(1.36-20.7)^{*}$ & $0.99(0.05-20.7)$ \\
\hline \multicolumn{7}{|l|}{ Birth order } \\
\hline 1 & 1.00 & 1.00 & 1.00 & 1.00 & 1.00 & 1.00 \\
\hline
\end{tabular}


Table 3 Univariate and multivariable logistic regression analyses of factors associated with early initiation of breastfeeding (Continued)

\begin{tabular}{|c|c|c|c|c|c|c|}
\hline \multirow[b]{2}{*}{ Characteristics } & \multicolumn{2}{|l|}{2000} & \multicolumn{2}{|l|}{ 2006-2007 } & \multicolumn{2}{|l|}{2013} \\
\hline & COR $(95 \% \mathrm{Cl})$ & AOR $(95 \% \mathrm{Cl})$ & COR (95\% Cl) & AOR $(95 \% \mathrm{Cl})$ & COR $(95 \% \mathrm{Cl})$ & AOR $(95 \% \mathrm{Cl})$ \\
\hline $2-3$ & $1.50(1.01-2.23)^{*}$ & $1.36(0.87-2.12)$ & $1.13(0.85-1.50)$ & $0.99(0.69-1.43)$ & $1.08(0.83-1.40)$ & $1.16(0.86-1.56)$ \\
\hline $4+$ & $1.10(0.74-1.64)$ & $1.20(0.67-2.15)$ & $1.21(0.86-1.72)$ & $1.23(0.78-1.95)$ & $1.42(1.03-1.97)^{*}$ & $1.52(1.03-2.26)^{*}$ \\
\hline \multicolumn{7}{|l|}{ Baby's birth size } \\
\hline Average & 1.00 & 1.00 & 1.00 & 1.00 & 1.00 & 1.00 \\
\hline Small & $0.39(0.24-0.63)^{* *}$ & $0.38(0.24-0.60)^{* *}$ & $0.64(0.44-0.93)^{*}$ & $0.63(0.44-0.90)^{*}$ & $0.71(0.51-0.99)^{*}$ & $0.72(0.65-1.01)$ \\
\hline Large & $0.55(0.37-0.79)^{*}$ & $0.51(0.34-0.78)^{*}$ & $0.67(0.51-0.90)^{*}$ & $0.69(0.51-0.94)^{*}$ & $0.83(0.64-1.07)$ & $0.86(0.52-1.13)$ \\
\hline
\end{tabular}

${ }^{a}$ Delivery mode was not available for the 2000 NDHS. However, this was measured in the later surveys

$A O R$ adjusted odds ratio, $C$ confidence interval, COR crude odds ratio (unadjusted odds ratio), NDHS Namibia Demographic Health Survey, TBAs traditional birth attendants ${ }^{* *} p$-value $<0.001{ }^{*} p$-value $<0.05$

services and health information in Namibia. However, our finding differs from reports from Ethiopia [13] and Ireland [50] that showed EIBF was more likely among employed women, and from Indonesia [12] where it was less likely among women from wealthy households.

\section{Strengths and limitations}

The use of publicly available, nationally representative data in this study allows our findings to be generalised to Namibia. However, our study has some limitations and caution is needed in interpreting the results. First, data collection for our main outcome relied on respondents' recall, meaning there is a likelihood of recall bias. Existing literature shows that overestimation or underestimation of EIBF is possible because of the mothers' inability to assess time in minutes or hours [51]. Moreover, it has been found that a mother's response to the question on when they first put their baby to the breast 'was related to the first time the newborn received breast milk rather than their first attempt to initiate breastfeeding' [51]. Second, data on delivery mode were not available before 2006-2007. Thus, we could not assess the trends and association of delivery mode and EIBF in 2000, but the later years showed a consistent pattern. Lastly, causality cannot be inferred as this was a crosssectional study.

\section{Policy and practice implications}

UNICEF and WHO are implementing a global initiative to improve breastfeeding outcomes with a goal of improving the average EIBF rate to $70 \%$ globally [1]. Namibia has achieved this target because of government commitment through the implementation of policies and programmes (e.g. Food and Nutrition Policy for Namibia, National Policy on Infant and Young Child Feeding, Food and Nutrition Guidelines) and a focus on accelerating the achievement of better child health indicators since 1993 [52]. However, the EIBF rate has declined over the recent years, eroding the gains of various programmes and policies. There is a need for increased focus on reviewing existing breastfeeding policies and ensuring full implementation of relevant breastfeeding policies and programmes such as BMFI to accelerate progress towards reversing this trend of declining EIBF in Namibia and contribute to achieving the sustainable development goals 3 on health.

\section{Conclusion}

Namibia experienced a declining trend in the EIBF rate from 2000 to 2013. Factors associated with EIBF also changed over the years. In 2000, urban residence, poorer women, ANC attendance and baby's birth size were associated with EIBF. Associated factors in 2013 were maternal age, marital status, caesarean section, TBA-assisted delivery, and birth order. These findings suggest there is a need for renewed commitment to promote breastfeeding in Namibia to reverse the trend of declining EIBF.

\section{Additional file}

Additional file 1: Figure S1. Flow diagram showing how the sample was obtained, provides information on the survey years, the number of households surveyed and the response rate both at the household level and for the women, detailing the number of births in the preceding 5 years, babies aged $<24$ months old and the singleton babies. (DOCX $69 \mathrm{~kb}$ )

\section{Abbreviations}

ANC: Antenatal care; AOR: Adjusted odds ratio; BMFI: Baby Mother-Friendly Initiative; Cl: Confidence interval; COR: Crude odds ratio; EIBF: Early initiation of breastfeeding; HIV: Human immunodeficiency virus; MDGs: Millennium development goals; NDHS: Namibia Demographic Health Survey; OR: Odds ratio; TBAs: Traditional birth attendants; UNICEF: United Nations Children's Fund; WHO: World Health Organisation

\section{Acknowledgements}

We are grateful to Dr. O'Brien Kyololo, Kevin Momanyi and Richard Kalisa for reviewing an earlier version of this paper, the Demographic and Health Survey Programme for allowing us access to the data and Audrey Holmes for editing and proofreading drafts of the manuscripts.

\section{Availability of data and materials}

The NDHS data and materials used in this study are available for free and on request on the Demographic and Health Survey website at www.dhsprogram.com. 


\section{Authors' contributions}

SMG, MNN, HMM and DCK conceptualised and designed the study; SMG obtained the data and MNN and SMG analysed and wrote the first draft. HMM and DCK interpreted the results and critically revised earlier drafts of the manuscript. SMG, MNN, HMM and DCK approved the last version of the manuscript and are accountable for all aspects of the work.

\section{Ethical approval and consent to participate}

Data used for this study were accessed through the Demographic and Health Survey website after completion of a user's agreement and granting of access. NDHS sought the necessary ethical approvals before survey data collection from the University of Namibia Research and Ethics Committee and the Human Research Committee of the ICF Macro International. All interviewed respondents gave informed consent and publicly available data were anonymised [18, 20, 21].

\section{Competing interests}

The authors declare that they have no competing interests.

\section{Publisher's Note}

Springer Nature remains neutral with regard to jurisdictional claims in published maps and institutional affiliations.

\section{Author details}

'Division of Social Medicine and Global Health, Department of Clinical Sciences, Lund University, Malmö, Sweden. ${ }^{2}$ School of Nursing and Midwifery, Aga Khan University, P.O. Box 30270 - 00100, Nairobi, Kenya. ${ }^{3}$ Kenya Red Cross Society, P.O. Box 40712 - 00100, Nairobi, Kenya. ${ }^{4}$ School of Medicine and Health Sciences, Kabarak University, P.O. Private Bag 20157, Kabarak, Kenya.

\section{Received: 25 June 2017 Accepted: 30 April 2018}

\section{Published online: 16 May 2018}

\section{References}

1. United Nations Children's Fund. World Health Organization: Global Breastfeeding Scorecard. In: 2017: Tracking Progress for Breastfeeding Policies and Programmes. New York: United Nations Children's Fund (UNICEF) and World Health Organization (WHO); 2017.

2. World Health Organization. Infant and young child feeding: a tool for assessing national practices, policies and programmes. Geneva: World Health Organization; 2003.

3. Tang L, Binns CW, Lee AH, Pan X, Chen S, Yu C. Low prevalence of breastfeeding initiation within the first hour of life in a rural area of Sichuan Province. Birth. 2013;40(2):134-42

4. Exavery A, Kante AM, Hingora A, Phillips JF. Determinants of early initiation of breastfeeding in rural Tanzania. Int Breastfeed J. 2015;10(1):27.

5. Victora CG, Bahl R, Barros AJD, França GVA, Horton S, Krasevec J, Murch S, Sankar M, Walker N, Rollins NC. Breastfeeding in the 21st century: epidemiology, mechanisms, and lifelong effect. Lancet. 2016;387(10017):475-90.

6. Eglash A, Montgomery A, Wood J. Breastfeeding. Dis Mon. 2008;54(6):343-411.

7. Debes AK, Kohli A, Walker N, Edmond K, Mullany LC. Time to initiation of breastfeeding and neonatal mortality and morbidity: a systematic review. BMC Public Health. 2013;13(Suppl 3):S19.

8. NEOVITA Study Group. Timing of initiation, patterns of breastfeeding, and infant survival: prospective analysis of pooled data from three randomised trials. Lancet Glob Health. 2016;4(4):e266-75.

9. Adewuyi EO, Zhao Y, Khanal V, Auta A, Bulndi LB. Rural-urban differences on the rates and factors associated with early initiation of breastfeeding in Nigeria: further analysis of the Nigeria demographic and health survey, 2013. Int Breastfeed J. 2017;12:51.

10. Takahashi K, Ganchimeg T, Ota E, Vogel JP, Souza JP, Laopaiboon M, Castro CP, Jayaratne K, Ortiz-Panozo E, Lumbiganon $P$, et al. Prevalence of early initiation of breastfeeding and determinants of delayed initiation of breastfeeding: secondary analysis of the WHO global survey. Sci Rep. 2017;7:44868

11. Tilahun G, Degu G, Azale T, Tigabu A. Prevalence and associated factors of timely initiation of breastfeeding among mothers at Debre Berhan town, Ethiopia: a cross-sectional study. Int Breastfeed J. 2016;11:27.

12. Titaley CR, Loh PC, Prasetyo S, Ariawan I, Shankar AH. Socio-economic factors and use of maternal health services are associated with delayed initiation and non-exclusive breastfeeding in Indonesia: secondary analysis of Indonesia demographic and health surveys 2002/2003 and 2007. Asia Pac J Clin Nutr. 2014;23(1):91-104.

13. Lakew $Y$, Tabar L, Haile D. Socio-medical determinants of timely breastfeeding initiation in Ethiopia: evidence from the 2011 nation wide demographic and health survey. Int Breastfeed J. 2015;10:24.

14. Berde AS, Yalcin SS. Determinants of early initiation of breastfeeding in Nigeria: a population-based study using the 2013 demograhic and health survey data. BMC Pregnancy Childbirth. 2016;16:32.

15. Liben ML, Yesuf EM. Determinants of early initiation of breastfeeding in Amibara district, northeastern Ethiopia: a community based cross-sectional study. Int Breastfeed J. 2016;11(1):7.

16. Namibia Ministry of Health and Social Services, United Nations Children's Fund Namibia. Towards a baby and mother friendly nation: Guidelines for implemention of the Baby and Mother Friendly Initiative. Windhoek: Ministry of Health and Social Services; 1992.

17. United Nations Children's Fund: Current status of baby-friendly hospital initiative march 2002. In. New York, United Nations Children's Fund; 2002.

18. Namibia Ministry of Health and Social Services. Namibia Demographic and Health Survey 2000. Windhoek: Ministry of Health and Social Services; 2003.

19. Namibia Ministry of Health and Social Services. Surveillance Report of the 2016 National HIV Sentinel Survey. Windhoek: Ministry of Health and Social Services; 2016.

20. Namibia Ministry of Health and Social Services. Namibia Demographic and Health Survey 2013. Windhoek and Rockville: Ministry of Health and Social Services and ICF International; 2014.

21. Namibia Ministry of Health and Social Services. Namibia Demographic and Health Survey 2006-07. Windhoek and Calverton: Ministry of Health and Social Services and ICF International; 2008.

22. World Health Organisation, United Nations Children's Fund. Global strategy for infant and young child feeding. Geneva: World Health Organisation and United Nations Children's Fund; 2003

23. Dennis C-L. Breastfeeding initiation and duration: a 1990-2000 literature review. J Obstet Gynecol Neonatal Nurs. 2002;31(1):12-32.

24. Alebel A, Dejenu G, Mullu G, Abebe N, Gualu T, Eshetie S. Timely initiation of breastfeeding and its association with birth place in Ethiopia: a systematic review and meta-analysis. Int Breastfeed J. 2017:12:44.

25. Adhikari M, Khanal V, Karkee R, Gavidia T. Factors associated with early initiation of breastfeeding among Nepalese mothers: further analysis of Nepal demographic and health survey, 2011. Int Breastfeed J. 2014;9(1):21.

26. Vieira TO, Vieira GO, Giugliani ER, Mendes CM, Martins CC, Silva LR. Determinants of breastfeeding initiation within the first hour of life in a Brazilian population: cross-sectional study. BMC Public Health. 2010;10(1):760.

27. Howe LD, Hargreaves JR, Huttly SR. Issues in the construction of wealth indices for the measurement of socio-economic position in low-income countries. Emerg Themes Epidemiol. 2008;5:3.

28. Khanal V, Scott JA, Lee AH, Karkee R, Binns CW. Factors associated with early initiation of breastfeeding in western Nepal. Int J Environ Res Public Health. 2015;12(8):9562-74.

29. Bui QT-T, Lee H-Y, Le AT-K, Van Dung D, Vu LT-H. Trends and determinants for early initiation of and exclusive breastfeeding under six months in Vietnam: results from the multiple Indicator cluster surveys, 2000-2011. Glob Health Action. 2016;9(1):29433.

30. Zhang Z. Model building strategy for logistic regression: purposeful selection. Ann Transl Med. 2016;4(6):111

31. West BT. Statistical and methodological issues in the analysis of complex sample survey data: practical guidance for trauma researchers. J Trauma Stress. 2008;21(5):440-7.

32. Yilmaz E, Yilmaz Z, Isik H, Gultekin IB, Timur H, Kara F, Kucukozkan T. Factors associated with breastfeeding initiation and exclusive breastfeeding rates in Turkish adolescent mothers. Breastfeed Med. 2016;

33. Kalisa R, Malande O, Nankunda J, Tumwine JK. Magnitude and factors associated with delayed initiation of breastfeeding among mothers who deliver in Mulago hospital, Uganda. Afr Health Sci. 2015;15(4):1130-5.

34. Wolde T, Chogo M, Bariso G, Dinka O, Abire O, Asfaw Se, Assen S, Ejeta E, Birhanu T, Nekemte E. Assessment of knowledge, attitude and practice of lactating mothers on complementary feeding in Jimma Arjo Town, Eastern Wollega Zone, Western Ethiopia. Assessment. 2015;36:71-7.

35. Kenya National Bureau of Statistics, Ministry of Health, National AIDS Control Council, Kenya Medical Research Institute, National Council for Population 
and Development, ICF International. Kenya Demographic and Health Survey 2014. Nairobi and Rockville: KNBS, MoH, NACC, KEMRI, NCPD and ICF; 2015.

36. World Health Organisation Regional Office for Africa. Issues and challenges Progress on the Health-Related MDGs. 2018. http://www.aho.afro.who.int/ profiles_information/index.php/Namibia:Issues_and_challenges___Progress_ on_the_Health-Related_MDGs. Accessed 1 Feb 2018.

37. World Health Organisation Regional Office for Africa. Accelerating achievement of MDGs 4 AND 5: PARMaCM launched in Namibia. 2013. http://www.afro.who.int/news/accelerating-achievement-mdgs-4-and-5parmacm-launched-namibia. Accessed 1 Feb 2018.

38. Wallwiener S, Muller M, Doster A, Plewniok K, Wallwiener CW, Fluhr H, Feller S, Brucker SY, Wallwiener M, Reck C. Predictors of impaired breastfeeding initiation and maintenance in a diverse sample: what is important? Arch Gynecol Obstet. 2016;294(3):455-66.

39. Watt S, Sword W, Sheehan D, Foster G, Thabane L, Krueger P, Landy CK. The effect of delivery method on breastfeeding initiation from the the Ontario mother and infant study (TOMIS) III. J Obstet Gynecol Neonatal Nurs. 2012; 41(6):728-37.

40. Albokhary AA, James JP. Does cesarean section have an impact on the successful initiation of breastfeeding in Saudi Arabia? Saudi Med J. 2014 35(11):1400.

41. Hobbs AJ, Mannion CA, McDonald SW, Brockway M, Tough SC. The impact of caesarean section on breastfeeding initiation, duration and difficulties in the first four months postpartum. BMC Pregnancy Childbirth. 2016;16:90.

42. Patel A, Bucher S, Pusdekar Y, Esamai F, Krebs NF, Goudar SS, Chomba E, Garces A, Pasha O, Saleem S. Rates and determinants of early initiation of breastfeeding and exclusive breast feeding at 42 days postnatal in six low and middle-income countries: a prospective cohort study. Reprod Health. 2015;12(2):S10

43. Tariku A, Alemu K, Gizaw Z, Muchie KF, Derso T, Abebe SM, Yitayal M, Fekadu A, Ayele TA, Alemayehu GA. Mothers' education and ANC visit improved exclusive breastfeeding in Dabat health and demographic surveillance system site, Northwest Ethiopia. PLoS One. 2017;12(6):e0179056.

44. Thu HN, Eriksson B, Khanh TT, Petzold M, Bondjers G, Kim CN, Thanh LN, Ascher $\mathrm{H}$. Breastfeeding practices in urban and rural Vietnam. BMC Public Health. 2012;12(1):964.

45. Mdala JF, Mash R. Causes of mortality and associated modifiable health care factors for children ( $<5$-years) admitted at Onandjokwe hospital, Namibia. Afr J Prim Health Care Fam Med. 2015;7(1):1-8.

46. Beyene MG, Geda NR, Habtewold TD, Assen ZM. Early initiation of breastfeeding among mothers of children under the age of 24 months in southern Ethiopia. Int Breastfeed J. 2016;12:1.

47. Sipsma HL, Magriples U, Divney A, Gordon D, Gabzdyl E, Kershaw T. Breastfeeding behavior among adolescents: initiation, duration, and exclusivity. J Adolesc Health. 2013;53(3):394-400.

48. Mossman M, Heaman M, Dennis CL, Morris M. The influence of adolescent mothers' breastfeeding confidence and attitudes on breastfeeding initiation and duration. J Hum Lact. 2008:24(3):268-77.

49. Adewuyi E, Adefemi K. Breastfeeding in Nigeria: a systematic review. Int J Commun Med Public Health. 2017:3(2):385-96.

50. Gallagher L, Begley C, Clarke M. Determinants of breastfeeding initiation in Ireland. Ir J Med Sci. 2016;185(3):663-8.

51. Salasibew MM, Filteau S, Marchant T. Measurement of breastfeeding initiation: Ethiopian mothers' perception about survey questions assessing early initiation of breastfeeding. Int Breastfeed J. 2014;9:13.

52. International Baby Food Action Network Geneva Infant Feeding Association (IBFAN-GIFA). Report on the situation of infant and young child feeding in Namibia. 2012. http://www.ibfan.org/art/CRC_61-Namibia-2012.pdf. Accessed 28 Jan 2018

\section{Ready to submit your research? Choose BMC and benefit from:}

- fast, convenient online submission

- thorough peer review by experienced researchers in your field

- rapid publication on acceptance

- support for research data, including large and complex data types

- gold Open Access which fosters wider collaboration and increased citations

- maximum visibility for your research: over $100 \mathrm{M}$ website views per year

At BMC, research is always in progress.

Learn more biomedcentral.com/submissions 